\title{
Application of Improved Chaotic Code in a Multi-User Coded Hybrid Spread Spectrum System under Frequency Selective Fading Channel
}

\author{
Hany A. A. Mansour*
}

\begin{abstract}
Spread spectrum techniques can be considered one of the main solutions to design a reliable and secure communication system. However, due to the developments of applications and huge increase of the demanded users, its applicable spreading codes become not sufficient to full fill all the security and applications requirements. As an alternative solution, a hybrid spread spectrum with application of specific codes appeared to solve most problems of the traditional spreading codes. Recently, many researches focused on the chaotic codes, due to its attractive security properties, in addition to its availability to generate a huge number of spreading codes. In this paper, the performance of a multi-user coded hybrid spread spectrum system is presented and evaluated under frequency selective fading channel. The system is constructed from conventional coding technique with Viterbi decoder, and three different types of spread spectrum technique using improved chaotic codes. The mathematical model is presented, and a performance evaluation and comparison is performed between different types of improved chaotic codes and the Gold code as spreading codes in the DSSS. The results show that, in the case of the multi-user, the improved chaotic codes give better performance than the Gold code.
\end{abstract}

Keywords: Spread Spectrum, Chaotic Code, Direct Sequence, Frequency Hopping, Time Hopping Spread Spectrum and Gold Sequence

\section{Introduction}

Generally, the security of the wireless communication channels had many challenges due to the developments of the interception and eavesdropping techniques. In addition, it is required to develop secure communication techniques capable of resist and mitigate the problems resultant from the channel impairments. One of the most powerful techniques used in this field are the spread spectrum (SS) techniques. The main idea of the spread spectrum relies upon expanding the bandwidth of the system much more than it actually requires, according to the SS technique [1]. The most popular types of SS are the direct sequence (DS), Frequency hopping (FH), time hopping (TH), chirp spread spectrum (CSS), and the hybrid spread spectrum (HSS). However, applying the spread spectrum techniques show some drawbacks, especially when the multiple access demands increased, and the spectrum allocations become dense [2,3]. One of the main problems of the spread spectrum techniques is the spreading codes, such as the traditional PN codes used in the DS technique, which becomes not sufficient to full fill all the development requirements. Although traditional PN spreading codes have attractive properties, nevertheless it has also many drawbacks such as 
the limitation of the generated sequences, in addition to its periodicity feature. These drawbacks make the intercepted signal predictable and can be reconstructed by linear regression which leads to security limitations. Chaotic codes can be considered as an alternative solution and had many interests in secure communication due to its many privileges over the traditional codes [4].

Actually, chaotic codes have a lot of attractive properties over the conventional PN sequences, especially from the security point of view. It is completely non-periodic, wide band, and more difficult to predict and to reconstruct. Moreover, Chaos is a deterministic, random-like process found in non-linear dynamical system, which is non-converging and bounded [5]. It has also a very sensitive dependence upon its initial condition and parameters, which gives the availability to generate theoretically an infinite number of spreading codes [6]. These properties make chaotic signals more difficult to intercept, and more secured to decode the information spreaded upon them [7]. Moreover, chaotic codes had many applications in Ultra Wideband (UWB) communications [8], and radar communications [9]. It was showed in many researchers that even one dimensional chaotic code has a high security level.

In this paper, the performance of a multiuser coded DS/FFH/TH hybrid spread spectrum system is presented, analyzed and evaluated under frequency selective fading channel. The system is constructed from conventional coding technique with Viterbi decoder, and three kinds of spread spectrum technique, which are the DSSS, Fast FHSS, in addition to THSS. The mathematical model of the presented system is driven for both the transmitter and the receiver. The performance comparison is performed using chaotic code and Gold codes as a spreading code in the DSSS. The chaotic codes used in this paper are the traditional logistic code, and improved versions of the traditional logistic codes, which are the zero mean (ZM), self-balanced (SB), and zero mean self-balanced (ZMSB). The results show that, in the case of the multi user, the chaotic codes outperforming the performance of the Gold code.

\section{Chaotic Maps}

In this section, the generation of the various chaotic codes types used in the DSSS is presented and explained. The section starts with basic traditional logistic code, and then the $\mathrm{ZM}, \mathrm{SB}$, and ZMSB are presented. The basic real value chaotic sequence considered with the DSSS type can be generated using the logistic map represented by equation 1 as:

$$
x_{i+1}=R x_{i}\left(1-x_{i}\right), \quad x_{i} \in(0,1)
$$

where, $x_{i+1}$ is the new value generated from the old value $x_{i}, R$ is the bifurcation parameter. To generate the basic ZM binary chaotic sequence, the real values of the basic chaotic sequence are mapped to the binary values. Actually, there are different methods used to map the real values of the chaotic code into the binary values [10]. In this paper, the chaotic mean sequence method will be considered as represented by equation 2 ,

$$
X_{1}=\operatorname{sign}(g\{x(t)-\operatorname{mean}[x(t)]\})
$$

This method depends on shifting the real value for the basic chaotic code by its mean value and generates a new basic sequence $\mathrm{g}(\mathrm{x})$ has a zero mean. The binary values are obtained by taking the sign function of the new zero mean sequence $\mathrm{g}(\mathrm{x})$. Finally, to generate basic SBZM chaotic sequence, the self-balancing process is performed in which the process is explained in detail in [11]. 


\section{Transmitter Model}

Figure 1 shows the whole construction of the multiuser hybrid spread spectrum transmitter system. Regarding to each user, the system is constructed from the baseband data unite, which generate data with default rate $\mathrm{C}_{1}=10 \mathrm{Kbps}$. The data generator is followed by encoder (error correcting code) unit, which generate error correcting code with code rate equal to 2 . The output of the encoder will be spreaded throughout the spreader unit which generates the spreading code PN1, The type of the spreading code is chaotic code generated form logistic map with spreading gain adjusted to be $\mathrm{C}_{3}=5 \mathrm{Mbps}$. After that the signal is modulated with the differential binary shift keying (DBSK) to eliminate the phase ambiguity occurred due to the rotation of the constellation as a result of some effect in the communications channel. This modulated signal has a bi-polarity transformation, which converts the data from 0,1 to be 1 , 1 respectively, to be able to be modulated by the frequency hopping set.

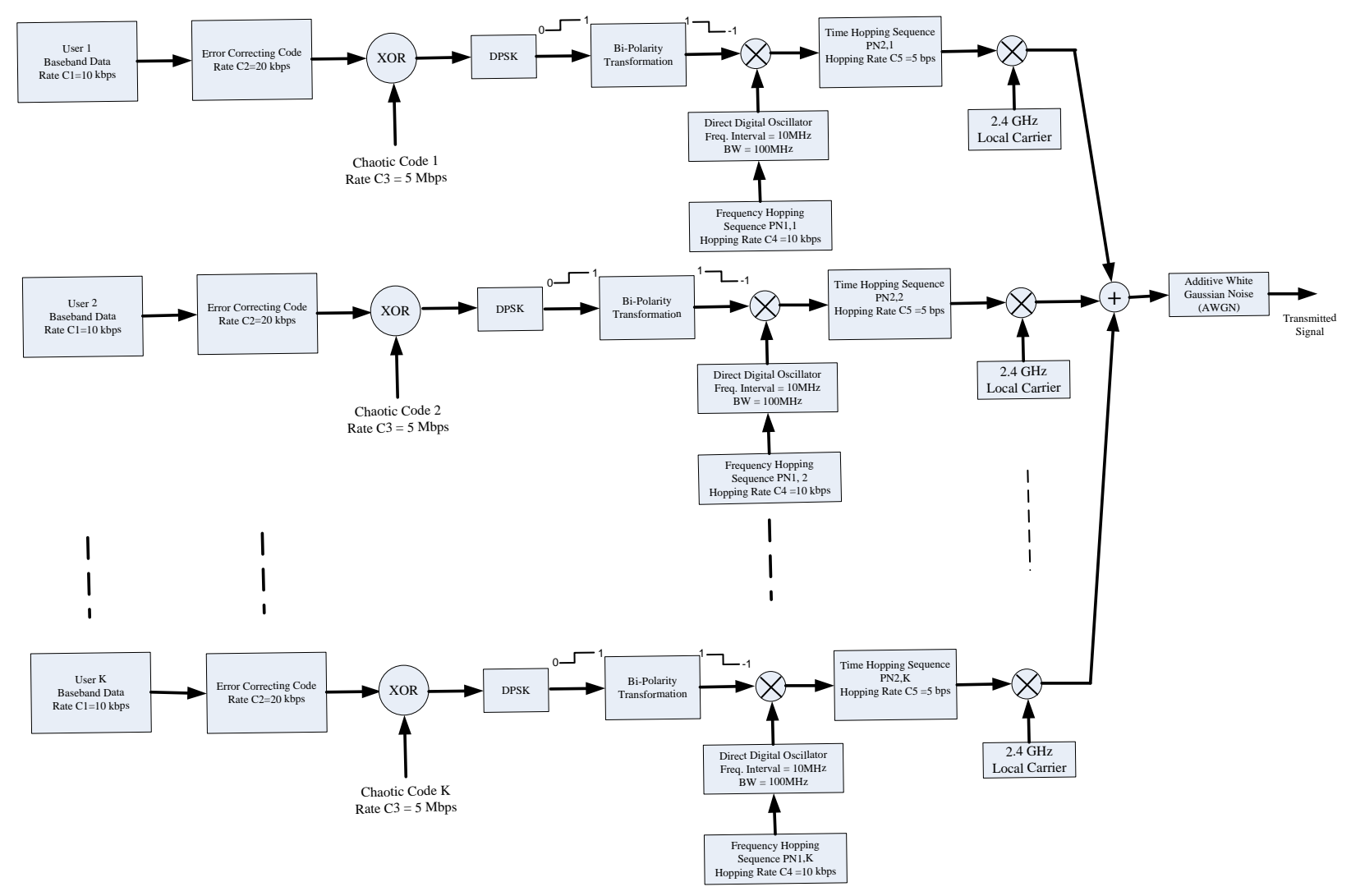

Fig. 1. Block Diagram of the Multi-User Hybrid Spread Spectrum Transmitter

The hopping frequencies are injected through the direct digital oscillator according to the pattern specified by the spreading code PN2. The hopping type is fast frequency hopping using 8 different frequencies, with hopping rate $100 \mathrm{Kbps}$, which means that each coded bit is modulated by 5 different frequencies. The hopping interval is set to be $10 \mathrm{MHZ}$, and the total transmitted bandwidth will be $100 \mathrm{MHZ}$. The frequencies are generated by the direct digital oscillator, and the hopping process is completely controlled throughout the sequence PN2.

Finally, the frequency hopped signal is transmitted in a certain time windows identified by the code PN3. The hopping rate is adjusted to be $5 \mathrm{bps}$, which mean that the time window is 200 ms by using the time hopping unite according to the pattern specified by the spreading code PN3 to the noisy channel. 
Actually, the system is not designed to use only one set of PN1, PN2, and PN3 codes, but the system is designed to deal with many groups of these codes called bank of parameters for each user. In the actual case the bank of these parameters or the number of the groups manipulated by the system is set to be 8 . This means that there are 8 different PN1 codes, 8 different PN2 codes (and correspondingly 8 different frequencies sets) and finally 8 different PN3 codes for each user. The parameter that identifies which group will be used is a frequency called the unified frequency constructed from 3 binary digits, this frequency is also sent to the receiver to unify the receiving parameters stored in the receiver during the receiving process.

In the case of the multiuser, the situation is more complicated, the system is designed to communicate with a large number of users at the same time. To keep the security level high as much as possible, the system is designed such that each user has its own codes library (or codes group), and consequently each user has its private unification factor. This private unification factor controls the spreading codes groups used in the direct sequence, frequency hopping, and time hopping operations. Since each user has a private unification factor, the set of the spreading codes for each user will be completely different from the other spreading codes set of all the other users. In addition, the unification factor specified to each user is completely independent on the other unification factors specified to the other users.

To identify the mathematical model of the given system, let the baseband data represented as:

$$
d(t)=\sum_{k=1}^{K} \sum_{i=0}^{M-1} x_{k}\left(t-i m T_{s}\right)
$$

where $\mathrm{K}$ expresses the total number of users, $\mathrm{M}$ is the number of the raw baseband data bits generated during the assigned time frame, $\mathrm{x}(\mathrm{t})$ is the rectangular pulse with duration $\mathrm{mT}_{\mathrm{s}}, \mathrm{m}$ is the number of samples in one-bit duration, and $\mathrm{T}_{\mathrm{s}}$ is the sampling time which equal to the inverse of sampling frequency $\mathrm{F}_{\mathrm{s}}$. The baseband data is the input to the conventional encoder which has an output can be represented as

$$
d_{c}(t)=\sum_{k=1}^{K} \sum_{i_{c}=0}^{M_{c}-1} x_{c k}\left(t-i_{c} m_{c} T_{s}\right)
$$

where $M_{c}$ is the number of coded bits generated during the same assigned time frame such that $\mathrm{M}_{\mathrm{c}}=2 \mathrm{M}, \mathrm{x}_{\mathrm{ck}}(\mathrm{t})$ is the coded rectangular pulse with duration $\mathrm{m}_{\mathrm{c}} \mathrm{T}_{\mathrm{s}}$ for the kth user, $\mathrm{m}_{\mathrm{c}}$ as previously mentioned, is the number of samples in one coded bit duration with $m_{c}=m / 2$.

As mentioned before, the spreading code used to make the direct sequence spread spectrum (PN1) is chaotic code, and since the coded data bit rate is $20 \mathrm{kbps}$ and the spreading sequence rate is $5 \mathrm{Mbps}$, so each coded data bit will be modulated with $\mathrm{N}=250$ different chips. This can be mathematically represented as:

$$
C(t)=\sum_{k=1}^{K} \sum_{i_{c}=o}^{M_{c}-1} \sum_{j=0}^{N-1} c_{k}\left(t-i_{c} N n T_{s}-j n T_{s}\right)
$$

where $\mathrm{C}(\mathrm{t})$ is the set of the chaotic code sequences for all the $\mathrm{K}$ users, $\mathrm{N}$ is the spreading gain which equal to 250 chip, $c_{k}(t)$ is the $k$ th user rectangular pulse (chip) with duration $\mathrm{nT}_{\mathrm{s}}, \mathrm{n}$ is the number of samples in one chip duration. Based on equation 5, the total spread signal can be represented as:

$$
S(t)=\sum_{k=1}^{K} \sum_{i_{c}=o}^{M_{c}-1} \sum_{j=0}^{N-1} z_{k}\left(t-n T_{s}\left[i_{c} N+j\right]\right)
$$

where $\mathrm{z}_{\mathrm{k}}(\mathrm{t})$ is also is the rectangular pulse with the same duration $\mathrm{nT}_{\mathrm{s}}$ of the kth user. The DS-FH signal in the case of using one group parameters can be represented as:

$$
S_{h}(t)=\sum_{k=1}^{K} \sum_{i_{c}=o}^{M_{c}-1} \sum_{j=0}^{N-1} z_{k}\left(t-n T_{s}\left[i_{c} N+j\right]\right) R e\left[\exp \left(J 2 \pi f_{k p} \frac{m_{c}}{G_{h}} l_{h} T_{s}\right)\right]
$$


where $f_{k p}$ is the kth user hopping frequency taking the values of $\left\{ \pm f_{k h}, \pm 2 f_{k h} \pm 3 f_{k h} \pm\right.$ $4 \mathrm{f}_{\mathrm{kh}}$, $\}$ according to the kth user controlled code sequence PN2, where $\mathrm{f}_{\mathrm{kh}}$ is the kth user frequency interval which set to be $10 \mathrm{MHz}$ as mentioned before. Also, the parameter $\mathrm{G}_{\mathrm{h}}$ can be considered as the hopping spreading gain, (in this case $G_{h}=5$ ). The time hopping process of the spreaded frequency hopping signal can be expressed considering only one group as:

$S_{t}(t)=\sum_{k=1}^{K} \sum_{i_{c}=o}^{M_{c}-1} \sum_{j=0}^{N-1} z_{k}\left(t-n T_{s}\left[i_{c} N+j\right]\right) R e\left[\exp \left(J 2 \pi f_{k p} \frac{m_{c}}{G_{h}} l_{h} T_{s}\right)\right] w_{k}\left(l_{t} T_{s}\right)$

where $\mathrm{w}_{\mathrm{k}}\left(\mathrm{l}_{\mathrm{t}} \mathrm{T}_{\mathrm{s}}\right)$ is the $k$ th user time window in which the signal will be transmitted. Finally, the noisy multi-user transmitted signal will take the expression of:

$S_{h t}(t)=\sum_{k=1}^{K} \sum_{i_{c}=0}^{M_{c}-1} \sum_{j=0}^{N-1} z_{k}\left(t-n T_{s}\left[i_{c} N+j\right]\right) \operatorname{Re}\left[\exp \left(J 2 \pi f\left(k_{g}\right) \frac{m_{c}}{G_{h}} l_{h} T_{s}\right)\right] W_{k_{g}}\left(l_{t} T_{s}\right)+n(t)$

where $n(t)$ represents the AWGN component.

\section{Receiver Model}

In this section, the receiver system block diagram shown in figure 2 is presented. The system is generally constructed from the blocks that perform the invers functions proceeded in the transmitter to retrieve the signal in addition to some other assistant blocks that help in the synchronization process and purifying the signal from the undesired noise and interferences.

For the multiuser fading channel, the impulse response of the fading channel can be represented as

$$
h(t)=\sum_{k=1}^{K} \sum_{l=1}^{L} \alpha_{k l} e^{j \varphi_{k l}} \delta\left(t-\tau_{k l}\right)
$$

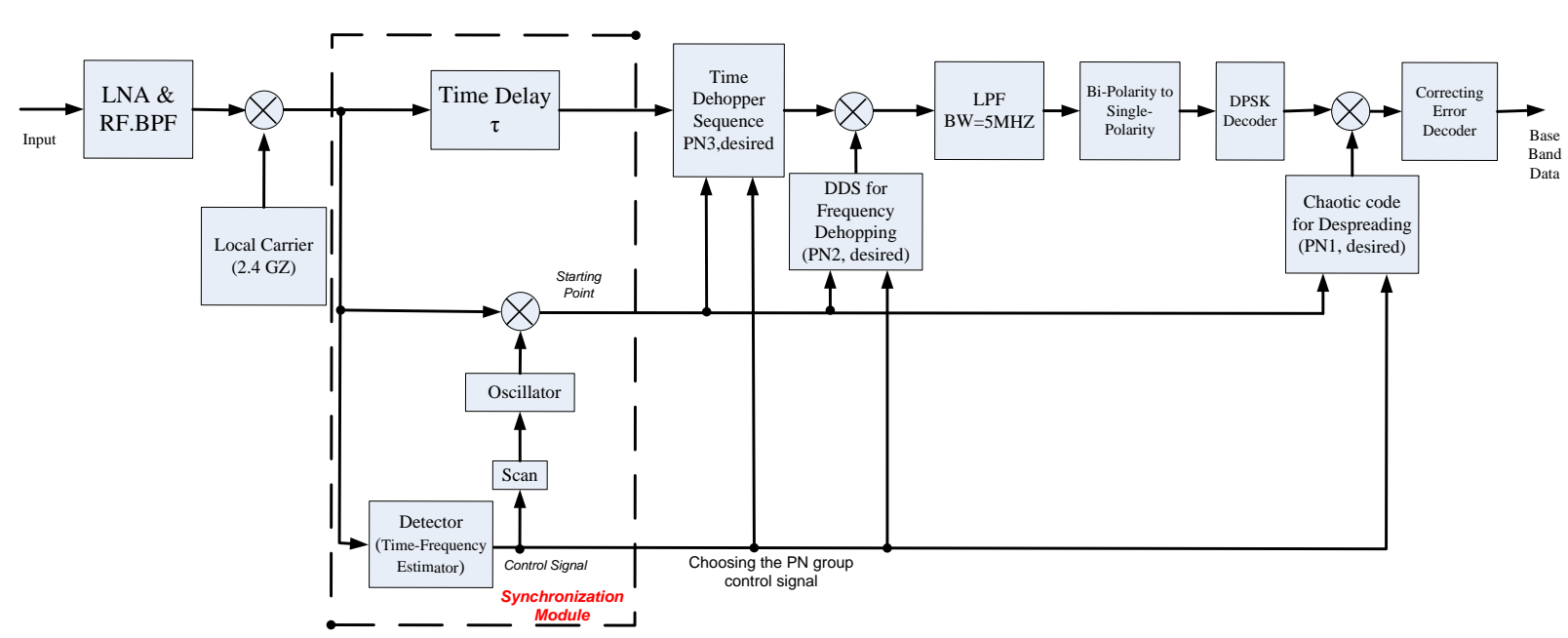

Fig. 2. Block Diagram of the Hybrid Spread Spectrum Receiver

where $\alpha_{k l}, \tau_{k l}$, and $\varphi_{k l}$ are the lth path gain, time delay, and phase of the $k t h$ user respectively, and $L$ is the total number of paths, assuming that all the users has the same number of paths. According to the impulse response of the multi-user fading channel illustrated in equation 10 , and in presence of AWGN with zero mean $(\mu=0)$ and 
variance $\sigma^{2}=N_{o} / 2, N_{o}$ is the noise power spectral density, the total received signal at the receiver can be represented as

$$
\begin{aligned}
R_{F}(t)=\sum_{k=1}^{K} \sum_{i_{c}=0}^{M_{c}-1} \sum_{l=1}^{L} \sum_{j=0}^{N-1} \alpha_{k l} e^{j \varphi_{k l} z_{k}\left(t-T_{s s}\left[i_{c} N+j\right]\right.} \\
\left.-\tau_{k l}\right) \operatorname{Re}\left[\exp \left(J 2 \pi f\left(k_{g}\right) \frac{m_{c}}{G_{h}} l_{h} T_{s}\right)\right] W_{k_{g}}\left(l_{t} T_{s}\right)+n(t)
\end{aligned}
$$

where $n(t)$ represents the AWGN component. According to the receiver structure in figurer 2 , the first block facing the received signal is the low noise amplifier, whose output can be expressed as

$$
\begin{aligned}
R_{F a}(t)=a \sum_{k=1}^{K} & \sum_{i_{c}=0}^{M_{c}-1} \sum_{l=1}^{L} \sum_{j=0}^{N-1} \alpha_{k l} e^{j \varphi_{k l}} z_{k}\left(t-T_{s s}\left[i_{c} N+j\right]\right. \\
& \left.-\tau_{k l}\right) \operatorname{Re}\left[\exp \left(J 2 \pi f\left(k_{g}\right) \frac{m_{c}}{G_{h}} l_{h} T_{s}\right)\right] W_{k_{g}}\left(l_{t} T_{s}\right)+\operatorname{an}(t)
\end{aligned}
$$

where $a$ is the amplification factor applied by the low noise amplifier. Performing the frequency down conversion process to eliminate the carrier and making the synchronization process, the resultant signal can be expressed as:

$$
\begin{aligned}
R_{F a}(t)=a \sum_{k=1}^{K} & \sum_{i_{c}=0}^{M_{c}-1} \sum_{l=1}^{L} \sum_{j=0}^{N-1} \alpha_{k l} e^{j \varphi_{k l} z_{k}}\left(t-T_{s s}\left[i_{c} N+j\right]\right. \\
& \left.-\tau_{k l}\right) \operatorname{Re}\left[\exp \left(J 2 \pi f_{k p} \frac{m_{c}}{G_{h}} l_{h} T_{s}\right)\right] W_{k_{g}}\left(l_{t} T_{s}\right)+\operatorname{an}(t)
\end{aligned}
$$

This equation represents the input multi-user fading signal to the time hopping gate. The output of this gate will take the form of

$$
\begin{aligned}
R_{F T H}(t)=a & \sum_{k=1}^{K} \sum_{i_{c}=0}^{M_{c}-1} \sum_{l=1}^{L} \sum_{j=0}^{N-1} \alpha_{k l} e^{j \varphi_{k l}} z_{k}\left(t-T_{s S}\left[i_{c} N+j\right]\right. \\
& \left.-\tau_{k l}\right) \operatorname{Re}\left[\exp \left(J 2 \pi f_{k p} \frac{m_{c}}{G_{h}} l_{h} T_{s}\right)\right]+\operatorname{an}(t) W_{1_{g}}\left(l_{t} T_{s}\right)
\end{aligned}
$$

which mean that the received signal is received in the time duration specified by the $W_{k}\left(l_{t} T_{s}\right)$ function, which defining which PN3 will be used according to the unified frequency as mentioned in the transmitter section. The following step is to make the dehopping process, assuming a complete synchronization; the dehoped signal can be expressed as:

$$
\begin{aligned}
R_{F F H}(t)=a & \sum_{k=1}^{K} \sum_{i_{c}=0}^{M_{c}-1} \sum_{l=1}^{L} \sum_{j=0}^{N-1} \alpha_{k l} e^{j \varphi_{k l}} z_{k}\left(t-T_{s s}\left[i_{c} N+j\right]-\tau_{k l}\right) \\
& +\operatorname{an}(t) W_{1_{g}}\left(l_{t} T_{s}\right) \operatorname{Re}\left[\exp \left(J 2 \pi f_{1 p} \frac{m_{c}}{G_{h}} l_{h} T_{s}\right)\right]
\end{aligned}
$$


This equation means that the original signal is recovered to its bi-polar spread form, while the noise spectrum component is frequency hopped on the frequency hopping pattern specified by the spreading code PN2. The despreaded form of the fading signal can be expressed as

$$
\begin{aligned}
R_{F D S}(t)=a & \sum_{k=1}^{K} \sum_{i_{c}=0}^{M_{c}-1} \sum_{l=1}^{L} \alpha_{k l} e^{j \varphi_{k l}} x_{k}\left(t-i_{c} m_{c} T_{s}-\tau_{k l}\right) \\
& +a \sum_{i_{c}=o}^{M_{c}-1} \sum_{j=0}^{N-1} n\left(t-i_{c} N n T_{s}-j n T_{s}\right) W_{1_{g}}\left(l_{t} T_{s}\right) \operatorname{Re}\left[\exp \left(J 2 \pi f_{1 p} \frac{m_{c}}{G_{h}} l_{h} T_{s}\right)\right]
\end{aligned}
$$

And finally, the error corrected decoded fading data signal can be formed as

$$
\begin{aligned}
& R_{F D C}(t)=a \sum_{i=0}^{M-1} \alpha_{11} e^{j \varphi_{11}} x_{1}\left(t-i m T_{s}-\tau_{11}\right)+\sum_{i=0}^{M-1} \sum_{l=2}^{L} \alpha_{1 l} e^{j \varphi_{1 l}} z_{1}\left(t-i m T_{s}-\tau_{1 l}\right) \\
& +a \sum_{k=2}^{K} \sum_{i=0}^{M-1} \sum_{l=1}^{L} V_{k l}\left(t-i m T_{s}-\tau_{k l}\right) \\
& +a \sum_{i=0}^{M-1} \sum_{j=0}^{N-1} n\left(t-2 i N n T_{s}-j n T_{s}\right) W_{1_{g}}\left(l_{t} T_{s}\right) \operatorname{Re}\left[\exp \left(J 2 \pi f_{1 p} \frac{m_{c}}{G_{h}} l_{h} T_{s}\right)\right]
\end{aligned}
$$

where

$$
V(t)=\alpha e^{j \varphi} Z(t)
$$

In equation 17, the first term represents the received signal from the main path of the first user, assuming that the first user is the desired user, while the second term expresses the received delayed signal versions from the remaining paths of the first user. The $V_{k l}$ showed in the third term represents all the fading paths for all the other interfering users (MAI), and finally the last term represents the noise component. In the third term, $V_{k l}$ are the delayed signals versions received from the all multipaths and despreaded by the assigned spreading code. The second and third terms are responsible about the enhancement difference occurred in the case of the AWGN and the fading channels. It is clear that the effect of these parts will be attenuated due to the cross correlation factors between the main spreading code and its shifted versions in the delayed signal versions, in addition to the frequency hopping effect.

\section{Simulation Results}

In this section, the performance of the presented system is presented and evaluated under the frequency selective fading channel. The fading channel is simulated with 5 paths has the following gain $\left[\begin{array}{lllll}0.8321 & 0.2774 & 0.2774 & 0.2774 & 0.2774\end{array}\right]$, with the corresponding paths delays 


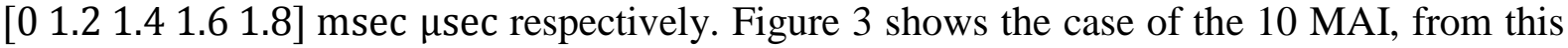
figure, it can be shown that all the chaotic codes nearly have identical performance, which means that the effect of low MAI is very small over the different chaotic codes. The figure shows also that the chaotic codes have generally a better resistance to the relatively low number of MAI than the Gold code under the frequency selective fading channel. The performance of the Gold code is degraded by about $3.5 \mathrm{~dB}$ at BER $10^{-4}$ due to the effect of the MAI under the frequency selective fading channel.

Figure 4 discusses the presented system performance when the MAI increased to 20 MAI under the same conditions. The results show that in this case, the Gold code performance is degraded due to the increasing of the interfering user's number, and achieves SNR $10 \mathrm{~dB}$ at BER $10^{-2}$. Generally, the performance of the chaotic codes are slightly affected by the increase of the MAI, however the performance of the SB chaotic code shows a little better performance.

Figure 5 represents the full capacity case, in which the MAI is increased to be 30 interfering users. The figure can show that the performance of the Gold code is completely degraded due the huge increase of the interfering user's number. It is clear also that the effect of the MAI in the frequency selective fading is increased on the chaotic logistic codes, however it still has much better performance over the Gold code performance, since it outperforming the Gold code performance by about $6 \mathrm{~dB}$ at BER $10^{-2}$.

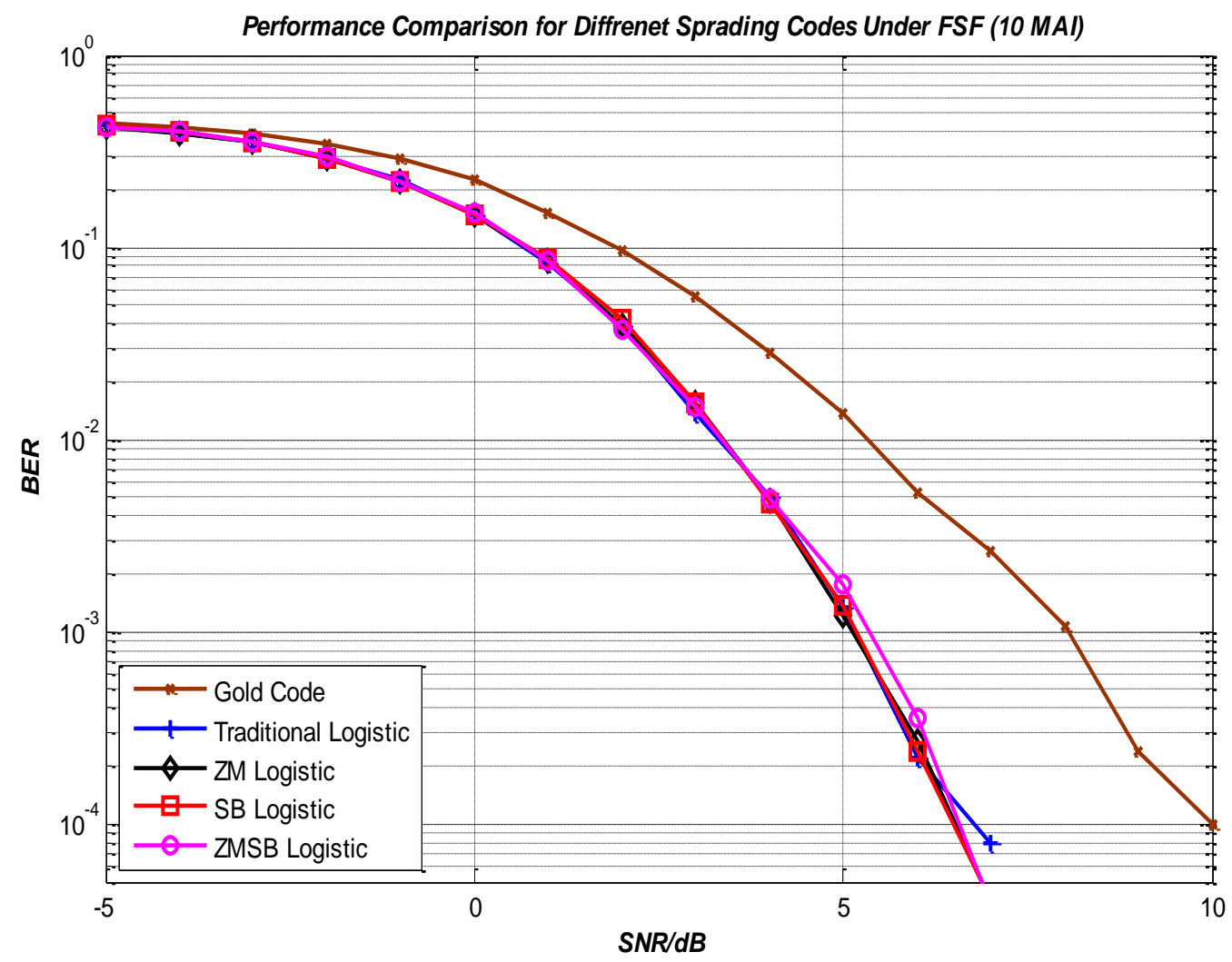

Fig. 3. Performance Comparison under Frequency Selective Fading Channel (10 MAI) 


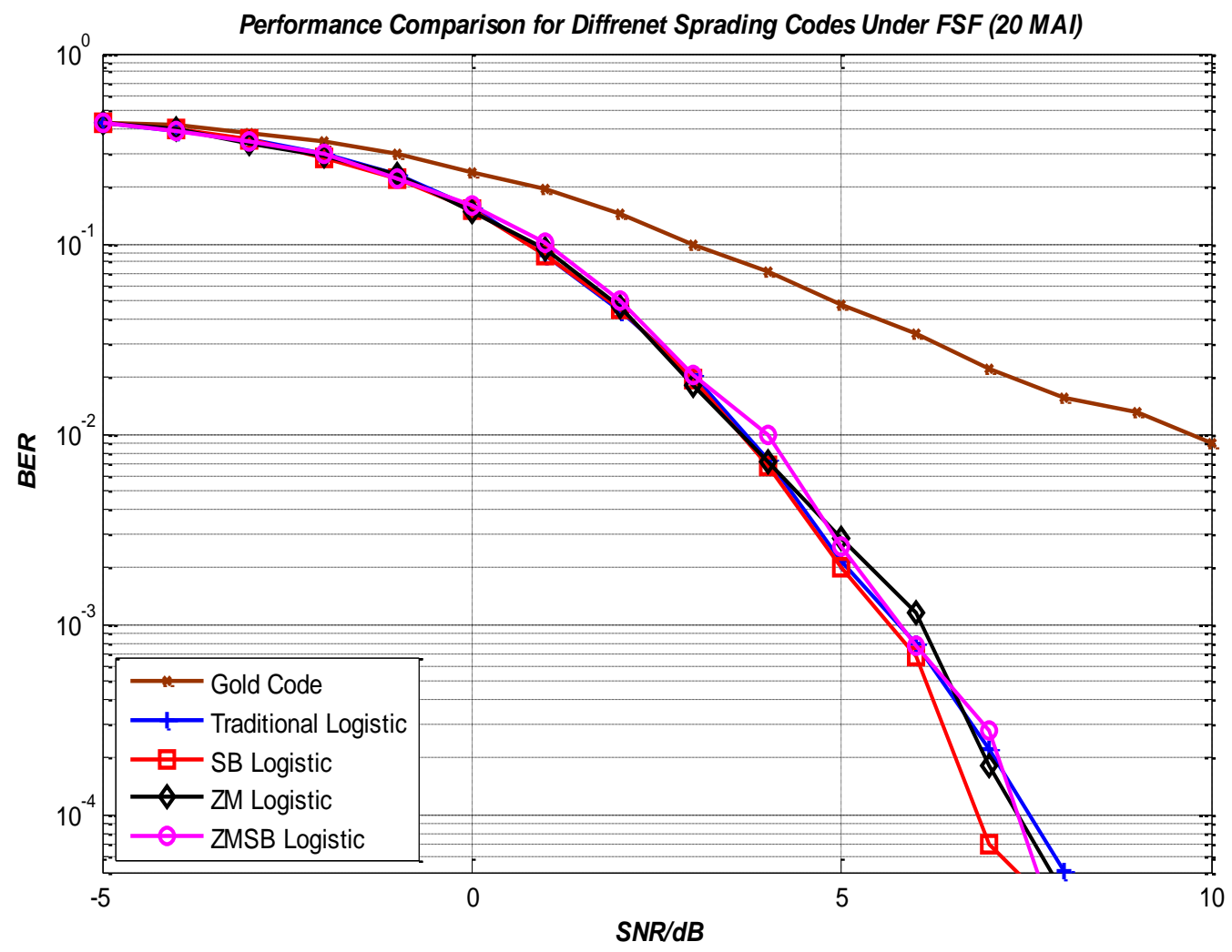

Fig. 4. Performance Comparison under Frequency Selective Fading Channel (20 MAI)

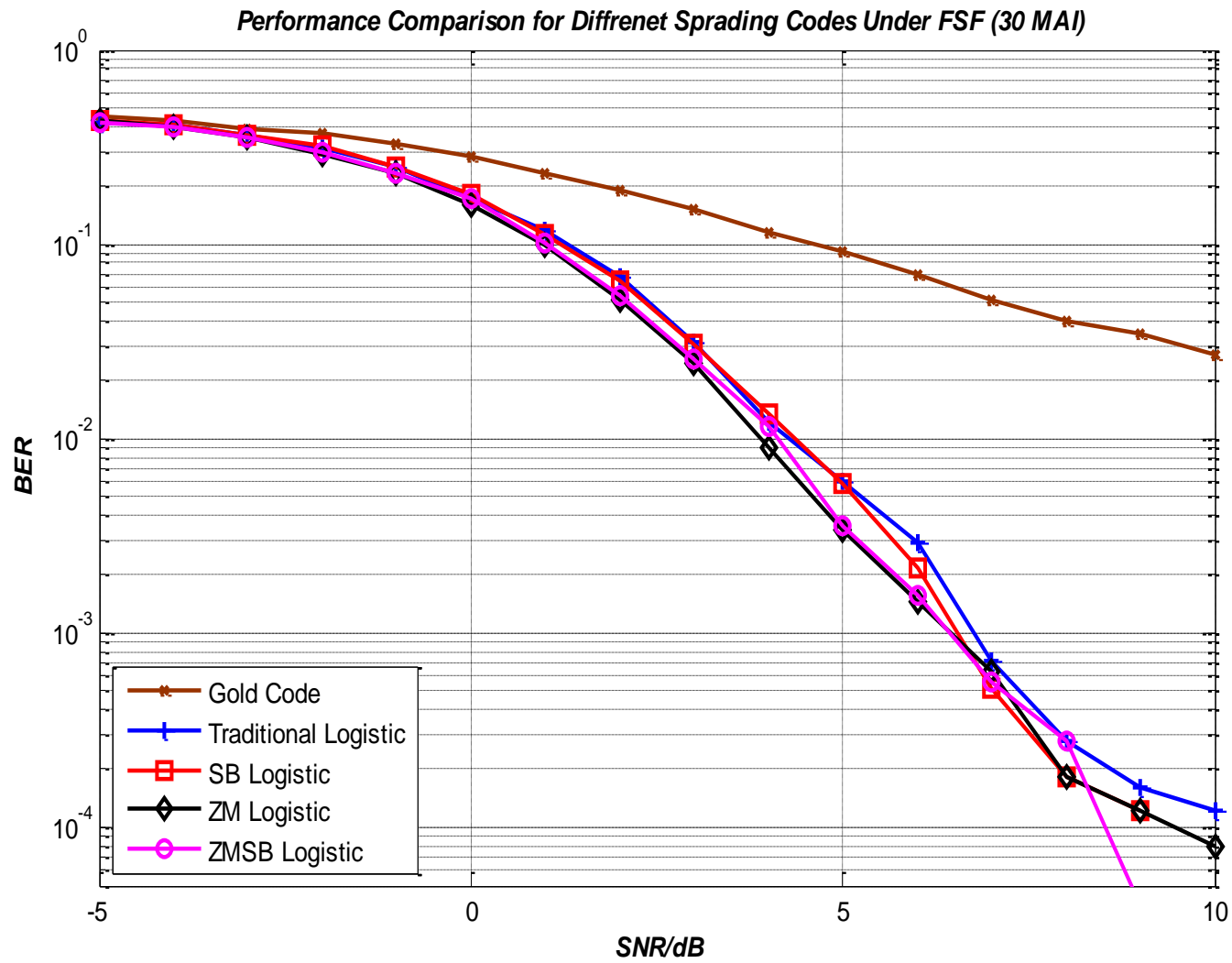

Fig. 5. Performance Comparison under Frequency Selective Fading Channel (30 MAI) 


\section{Conclusion}

This paper deals with the performance evaluation of the multi-user coded DS/FFH/TH hybrid spread spectrum system. The performance evaluation is performed using 4 different improved chaotic codes as spreading codes in the DSSS and compared with the performance of the Gold code. The Paper is started with an introduction, which gives an overview about the total system and its general idea. The mathematical model of the transmitter and receiver systems are discussed and derived in the case of the multi-user under frequency selective fading channel. The simulation is performed under different number of the MAI. The results show that the chaotic codes have generally better performance than the Gold code, especially when the number of the interfering is increased under the fading conditions.

\section{References}

[1] T.L. Carrolla, "Adaptive chaotic maps for identification of complex targets", IET Radar Sonar Navig. 2 (8) (2008) 256-262.

[2] Rajni Billa, "Analysis of Direct Sequence and Chirp Spread Spectrum over Flat Fading Channel for Multiple Access", IJCEM Vol. 15 Issue 5, September 2012.

[3] Rahul N, Amit T, "DS-SS Communication system using pseudo chaotic sequences generator", International Conference on Communication Systems and Network Technologies 2013.

[4] Lwaa Faisal Abdulameer, Jokhakar Jignesh D., U. Sripati , Murlidhar Kulkarni, "BER Performance Enhancement for Secure Wireless Communication Systems based on Chaotic-MIMO Techniques" International Conference on Communication and Electronics System Design, edited by M. Salim, K. K. Sharma, V. Janyani, Proc. of SPIE Vol. 8760, 87600VC 2013 SPIE - CCC code: 0277-786/13/\$18 • doi: 10.1117/12.2011155

[5] T. S. Rappaport, "Wireless Communications: Principles and Practice". Prentice Hall PTR, NJ, 2009.

[6] Henrieta P, "The type of chaotic sequences for signal transmission", SCYR - 10th Scientific Conference of Young Researchers - FEI TU of Košice 2010.

[7] R. Vali, S. Berber, and S. K. Nguang, "Accurate derivation of chaos-based acquisition performance in a fading channel", IEEE Trans. Wireless Commun., vol. 11, no. 2, pp. 722-731, Feb 2012.

[8] C.C. Chong, S.K. Yong, UWB direct chaotic communication technology for low-rate WPAN applications, IEEE Trans. Vehicular Technol. 57 (5) (2008) 1527-1536.

[9] Anamika Sarma, Kandarpa Kumar Sarma, Nikos Mastorakis, "Orthogonal Chaotic Sequence for Use in Wireless Channels' International Journal of Computers and Communications Volume 9, 2015

[10] G. V. REDDY, "Performance Evaluation of Different DS-CDMA Receivers Using Chaotic Sequences" Master Thesis, 2007.

[11] A. Yong, M. Rongzeng, C. Xiaolin, Y. Yeupeng “An Improved Method of Generating the Self-Balanced Chaotic Spread-Spectrum Code "High Technology Letters vol. 17 No. 1 pp. 46-51 Mar. 2011. 